\title{
Approximation by Baskakov-Szász-Stancu Operators Preserving Exponential Functions
}

\author{
Murat Bodur*, Övgü GÜrel YilmaZ, AND Ali Aral
}

\begin{abstract}
The purpose of this paper is to construct a general class of operators which has known BaskakovSzász-Stancu that preserving constant and $e^{2 a x}, a>0$ functions. We scrutinize a uniform convergence result and analyze the asymptotic behavior of our operators, as well. Finally, we discuss the convergence of corresponding sequences in exponential weighted spaces and make a comparison about which one approximates better between classical Baskakov-Szász-Stancu operators and the recent operators.
\end{abstract}

Keywords: Baskakov-Szász-Stancu operators, Exponential functions, Quantitative results, Weighted approximation. 2010 Mathematics Subject Classification: 41A25, 41A36.

\section{INTRODUCTION}

Approximation theory is one of the crucial subjects that is used by researchers. It is separated into many fields one of which is positive linear operators that play a key role in approximation theory. It has been the inspiration for so many mathematicians from the past. For years, many publications related to the approximation theory has made and has still being studied, too.

One of the remarkable work has been done related to the positive linear operators from King [16] in 2003. King described the modified Bernstein operators which preserve for $e_{i}(t)=t^{i}$, $i=0,2$ test functions and examined their approximation properties. He accomplished to take an attention in a short time from researchers who perform approximation theory. Since that time, lots of researchers have put forth many relevant studies on this issue. Numerous articles can be given interrelated with Kings research ( [4-7], [9], [18]).

In 2016 Acar et al. [1] investigated approximation properties of Szász-Mirakyan operators which preserving constant and $e^{2 a x}, a>0$. In that paper the rate of convergence of this generalization was obtained by means of the modulus of continuity. They also presented and proved theorems related on shape preserving properties.

Later this idea was applied to some other well known linear positive operators, such as Szász-Durrmeyer [8], Szász-Mirakyan-Kantorovich [13], Baskakov-Szász-Mirakyan [12], Philips Operators [14], Baskakov Operators [19] and the subject is still continue to be relevant.

In this study, inspired by the main paper [1], we constructed a new family of linear positive operators by using Baskakov-Szász-Stancu operators which is based on preserving exponential functions. 


\section{CONSTRUCTION OF THE Operators}

For $f \in C[0, \infty)$ and $k, n \in \mathbb{N}$ the Baskakov-Szász type operators was proposed by Gupta and Srivastava [?] as,

$$
M_{n}(f ; x)=n \sum_{k=0}^{\infty}\left(\begin{array}{c}
n+k-1 \\
k
\end{array}\right) \frac{x^{k}}{(1+x)^{n+k}} \int_{0}^{\infty} e^{-n t} \frac{(n t)^{k}}{k !} f(t) d t .
$$

In 2015, Mishra et al. considered Stancu type generalization of Baskakov-Szász operators [17] like as,

$$
B_{n}^{\alpha, \beta}(f ; x)=n \sum_{k=0}^{\infty}\left(\begin{array}{c}
n+k-1 \\
k
\end{array}\right) \frac{x^{k}}{(1+x)^{n+k}} \int_{0}^{\infty} e^{-n t} \frac{(n t)^{k}}{k !}(t) f\left(\frac{n t+\alpha}{n+\beta}\right) d t .
$$

Here, two parameters $\alpha$ and $\beta$ satify the condition $0 \leq \alpha \leq \beta$. We consider the following modified form of Baskakov-Szász-Stancu operators

$$
M_{n}^{\alpha, \beta}(f ; x)=n \sum_{k=0}^{\infty}\left(\begin{array}{c}
n+k-1 \\
k
\end{array}\right) \frac{\left(\theta_{n}(x)\right)^{k}}{\left(1+\theta_{n}(x)\right)^{n+k}} \int_{0}^{\infty} e^{-n t} \frac{(n t)^{k}}{k !} f\left(\frac{n t+\alpha}{n+\beta}\right) d t .
$$

We interest in investigated operators preserving $e_{0}$ and $e^{2 a x}$. Suppose these operators (2.2) preserve $e^{2 a x}$, then

$$
\begin{aligned}
M_{n}^{\alpha, \beta}\left(e^{2 a t} ; x\right) & =n \sum_{k=0}^{\infty}\left(\begin{array}{c}
n+k-1 \\
k
\end{array}\right) \frac{\left(\theta_{n}(x)\right)^{k}}{\left(1+\theta_{n}(x)\right)^{n+k}} \int_{0}^{\infty} e^{-n t} \frac{(n t)^{k}}{k !} e^{2 a(n t+\alpha) / n+\beta} d t \\
& =n e^{2 a \alpha / n+\beta} \sum_{k=0}^{\infty}\left(\begin{array}{c}
n+k-1 \\
k
\end{array}\right) \frac{\left(\theta_{n}(x)\right)^{k}}{\left(1+\theta_{n}(x)\right)^{n+k}} \frac{n^{k}}{k !} \int_{0}^{\infty} e^{-n t(n+\beta-2 a) /(n+\beta)} t^{k} d t \\
& =\frac{n+\beta}{n+\beta-2 a} e^{2 a \alpha / n+\beta} \sum_{k=0}^{\infty}\left(\begin{array}{c}
n+k-1 \\
k
\end{array}\right)\left(\frac{(n+\beta) \theta_{n}(x)}{n+\beta-2 a}\right)^{k}\left(\frac{1}{1+\theta_{n}(x)}\right)^{n+k} \\
& =\frac{n+\beta}{n+\beta-2 a} e^{2 a \alpha / n+\beta}\left(\theta_{n}(x)\left(1-\frac{n+\beta}{n+\beta-2 a}\right)+1\right)^{-n} .
\end{aligned}
$$

Taking into account $M_{n}^{\alpha, \beta}\left(e^{2 a t} ; x\right)=e^{2 a x}$, then we can find without hesitation

$$
\theta_{n}(x)=\frac{n+\beta-2 a}{2 a}\left(1-\left(\frac{n+\beta-2 a}{n+\beta} e^{2 a(x(n+\beta)-\alpha) / n+\beta}\right)^{-1 / n}\right) .
$$

Seemingly the function which is demonstrated $\theta_{n}(x)$ satisfies the situtation

$$
\theta_{n}(x)=\left(M_{n}^{\alpha, \beta}\left(e^{2 a t} ; x\right)\right)^{-1} \circ e^{2 a x} .
$$

\section{Auxiliary Results}

In this part, we shall present the moments and the central moments of the operators (2.2) which will be necessary to prove our main results. 
Lemma 3.1. Let $e_{i}(t):=t^{i}, i=0,1,2$. Then the Baskakov-Szász-Stancu operators $M_{n}^{\alpha, \beta}$ satisfies

$$
\begin{aligned}
& M_{n}^{\alpha, \beta}\left(e_{0} ; x\right)=1, \\
& M_{n}^{\alpha, \beta}\left(e_{1} ; x\right)=\frac{n \theta_{n}(x)+1+\alpha}{n+\beta}, \\
& M_{n}^{\alpha, \beta}\left(e_{2} ; x\right)=\frac{n(n+1)}{(n+\beta)^{2}}\left(\theta_{n}(x)\right)^{2}+\frac{4 n+2 n \alpha}{(n+\beta)^{2}} \theta_{n}(x)+\frac{2+2 \alpha+\alpha^{2}}{(n+\beta)^{2}} .
\end{aligned}
$$

Lemma 3.2. Let $\mu_{n, r}^{\alpha, \beta}(x)=M_{n}^{\alpha, \beta}\left((t-x)^{r}, x\right), r=0,1,2, \ldots$ Then by considering Lemma (3.1), we have

$$
\begin{aligned}
\mu_{n, 0}^{\alpha, \beta} & =1, \\
\mu_{n, 1}^{\alpha, \beta} & =\frac{n \theta_{n}(x)+1+\alpha}{n+\beta}-x, \\
\mu_{n, 2}^{\alpha, \beta} & =\frac{n(n+1)}{(n+\beta)^{2}}\left(\theta_{n}(x)\right)^{2}+\frac{4 n+2 n \alpha}{(n+\beta)^{2}} \theta_{n}(x)+\frac{2+2 \alpha+\alpha^{2}}{(n+\beta)^{2}}-2 x \frac{n \theta_{n}(x)+1+\alpha}{n+\beta}+x^{2} .
\end{aligned}
$$

Respectively, limits hold

$$
\lim _{n \rightarrow \infty} n\left(\frac{n \theta_{n}(x)+1+\alpha}{n+\beta}-x\right)=-a x(x+2)
$$

and

$$
\lim _{n \rightarrow \infty} n\left(\left(\frac{n \theta_{n}(x)+1+\alpha}{n+\beta}-x\right)^{2}+\left(\frac{n \theta_{n}(x)}{n+\beta}\right)^{2}+2 n \theta_{n}(x)+1\right)=x(x+2) .
$$

\section{MAIN RESULTS}

In this main section, we would like to show that the constructed operators are meticulously discussed linked with a uniform convergence result and a quantitative estimate. We debate the convergence of corresponding sequences in exponential weighted spaces.

Here, we will take $C^{*}[0, \infty)$ the class of real-valued continuous functions $f$, possessing finite limit for $x$ sufficiently large and equipped with the uniform norm.

Theorem A. [15] Take into account a sequence of positive linear operators $L_{n}: C^{*}[0, \infty) \rightarrow C^{*}[0, \infty)$ and set

$$
\begin{gathered}
\left\|L_{n}\left(e_{0}\right)-1\right\|_{[0, \infty)}=\alpha_{n}^{*}, \\
\| L_{n}\left(e^{-t}\right)-\left.e^{-x}\right|_{[0, \infty)}=\beta_{n}^{*}, \\
\| L_{n}\left(e^{-2 t}\right)-\left.e^{-2 x}\right|_{[0, \infty)}=\gamma_{n}^{*},
\end{gathered}
$$

then for each $f \in C^{*}[0, \infty)$

$$
\left\|L_{n}(f)-f\right\|_{[0, \infty)} \leq \alpha_{n}^{*}\|f\|_{[0, \infty)}+\left(2+\alpha_{n}^{*}\right) \omega^{*}\left(f ; \sqrt{\alpha_{n}^{*}+2 \beta_{n}^{*}+\gamma_{n}^{*}}\right) .
$$

where the modulus of continuity is defined as

$$
\omega^{*}(f ; \delta):=\sup _{\substack{x, t \geq 0 \\\left|e^{-x}-e^{-t}\right| \leq \delta}}|f(t)-f(x)| .
$$

Now, we can apply Theorem A for Baskakov-Szász-Stancu operators. 
Theorem 4.1. For each function $f \in C^{*}[0, \infty)$, we possess

$$
\left\|M_{n}^{\alpha, \beta} f-f\right\|_{[0, \infty)} \leq 2 \omega^{*}\left(f ; \sqrt{2 \beta_{n}^{*}+\gamma_{n}^{*}}\right),
$$

where

$$
\begin{gathered}
\left\|M_{n}^{\alpha, \beta}\left(e^{-t}\right)-e^{-x}\right\|_{[0, \infty)}=\beta_{n}^{*}, \\
\left\|M_{n}^{\alpha, \beta}\left(e^{-2 t}\right)-e^{-2 x}\right\|_{[0, \infty)}=\gamma_{n}^{*} .
\end{gathered}
$$

Proof. According the definition of the operators, since they preserve the constants, we reach

$$
\left\|M_{n}^{\alpha, \beta}\left(e_{0}\right)-1\right\|_{[0, \infty)}=\alpha_{n}^{*}=0
$$

and for $f(t)=e^{-t}$, we get

$$
\begin{aligned}
M_{n}^{\alpha, \beta}\left(e^{-t} ; x\right) & =n \sum_{k=0}^{\infty}\left(\begin{array}{c}
n+k-1 \\
k
\end{array}\right) \frac{\left(\theta_{n}(x)\right)^{k}}{\left(1+\theta_{n}(x)\right)^{n+k}} \int_{0}^{\infty} e^{-n t} \frac{(n t)^{k}}{k !} e^{-(n t+\alpha) / n+\beta} d t \\
& =\frac{n+\beta}{n+\beta+1} e^{-\alpha / n+\beta} \sum_{k=0}^{\infty}\left(\begin{array}{c}
n+k-1 \\
k
\end{array}\right)\left(\frac{(n+\beta) \theta_{n}(x)}{n+\beta+1}\right)^{k}\left(\frac{1}{1+\theta_{n}(x)}\right)^{n+k} \\
& =\frac{n+\beta}{n+\beta+1} e^{-\alpha / n+\beta}\left(\frac{\theta_{n}(x)}{n+\beta+1}+1\right)^{-n} .
\end{aligned}
$$

Using Maple to make a calculation of the right hand side which is found (4.4), we observe

$$
\begin{gathered}
M_{n}^{\alpha, \beta}\left(e^{-t} ; x\right)=e^{-x}+\frac{e^{-x}(2 a+1)\left(x^{2}+2 x\right)}{2 n}+\mathcal{O}\left(\frac{1}{n^{2}}\right), \\
\left\|M_{n}^{\alpha, \beta}\left(e^{-t}\right)-e^{-x}\right\|_{[0, \infty)}=\frac{2(2 a+1)}{n e^{2}}+\frac{2 a+1}{n e}+\mathcal{O}\left(\frac{1}{n^{2}}\right)=\beta_{n}^{*} .
\end{gathered}
$$

Also, for $f(t)=e^{-2 t}$, we have

$$
\begin{aligned}
M_{n}^{\alpha, \beta}\left(e^{-2 t} ; x\right) & =n \sum_{k=0}^{\infty}\left(\begin{array}{c}
n+k-1 \\
k
\end{array}\right) \frac{\left(\theta_{n}(x)\right)^{k}}{\left(1+\theta_{n}(x)\right)^{n+k}} \int_{0}^{\infty} e^{-n t} \frac{(n t)^{k}}{k !} e^{-2(n t+\alpha) / n+\beta} d t \\
& =\frac{n+\beta}{n+\beta+2} e^{-2 \alpha / n+\beta} \sum_{k=0}^{\infty}\left(\begin{array}{c}
n+k-1 \\
k
\end{array}\right)\left(\frac{(n+\beta) \theta_{n}(x)}{n+\beta+2}\right)^{k}\left(\frac{1}{1+\theta_{n}(x)}\right)^{n+k} \\
& =\frac{n+\beta}{n+\beta+2} e^{-2 \alpha / n+\beta}\left(\frac{2 \theta_{n}(x)}{n+\beta+2}+1\right)^{-n} .
\end{aligned}
$$

If the procedure applied for previous equality is performed again, we receive

$$
\begin{gathered}
M_{n}^{\alpha, \beta}\left(e^{-2 t} ; x\right)=e^{-2 x}+\frac{e^{-2 x}(a+1)\left(2 x^{2}+4 x\right)}{n}+\mathcal{O}\left(\frac{1}{n^{2}}\right), \\
\left\|M_{n}^{\alpha, \beta}\left(e^{-2 t}\right)-e^{-2 x}\right\|_{[0, \infty)}=\frac{2(a+1)}{n}\left(\frac{1}{e^{2}}+\frac{1}{e}\right)+\mathcal{O}\left(\frac{1}{n^{2}}\right)=\gamma_{n}^{*} .
\end{gathered}
$$

Here, $\beta_{n}^{*}$ and $\gamma_{n}^{*}$ tend to zero as $n \rightarrow \infty$ so this completes the proof.

Now, we will analyze the asymptotic behavior of given operators $M_{n}^{\alpha, \beta}$ with Voronovskaya-type theorem. 
Theorem 4.2. Let $f, f^{\prime \prime} \in C^{*}[0, \infty)$ then for any $x \in[0, \infty)$ we have

$$
\begin{aligned}
& \left|n\left[M_{n}^{\alpha, \beta}(f ; x)-f(x)\right]+a x(x+2) f^{\prime}(x)-\frac{x(x+2)}{2} f^{\prime \prime}(x)\right| \\
\leq & \left|p_{n}\right|\left|f^{\prime}(x)\right|+\left|q_{n} \| f^{\prime \prime}(x)\right|+2\left(2 q_{n}+x(x+2)+r_{n}\right) \omega^{*}\left(f^{\prime \prime}, \frac{1}{\sqrt{n}}\right),
\end{aligned}
$$

where

$$
\begin{aligned}
p_{n} & =n \mu_{n, 1}^{\alpha, \beta}(x)+a x(x+2), \\
q_{n} & =2^{-1}\left(n \mu_{n, 2}^{\alpha, \beta}(x)-x(x+2)\right), \\
r_{n} & =n^{2} \sqrt{M_{n}^{\alpha, \beta}\left(\left(e^{-x}-e^{-t}\right)^{4} ; x\right)} \sqrt{\mu_{n, 4}^{\alpha, \beta}(x)} .
\end{aligned}
$$

Proof. By Taylor's expansion of $f$ for some fixed $x$,

$$
f(t)=f(x)+f^{\prime}(x)(t-x)+\frac{1}{2} f^{\prime \prime}(x)(t-x)^{2}+h(t, x)(t-x)^{2},
$$

where

$$
h(t, x)=\frac{f^{\prime \prime}(\eta)-f^{\prime \prime}(x)}{2}
$$

and $\eta$ is a number lying between $x$ and $t$. Applying $M_{n}^{\alpha, \beta}$ to both sides of the above identity and using the linearity of the operators and Lemma (3.2), we get

$$
\begin{aligned}
& \left|n\left[M_{n}^{\alpha, \beta}(f ; x)-f(x)\right]+a x(x+2) f^{\prime}(x)-\frac{x(x+2)}{2} f^{\prime \prime}(x)\right| \\
\leq & \left|p_{n}\right|\left|f^{\prime}(x)\right|+\left|q_{n}\right|\left|f^{\prime \prime}(x)\right|+\left|n M_{n}^{\alpha, \beta}\left(h(t, x)(t-x)^{2} ; x\right)\right| .
\end{aligned}
$$

Obviously it is enough to consider the last term of the inequality $\left|n M_{n}^{\alpha, \beta}\left(h(t, x)(t-x)^{2} ; x\right)\right|$. Taking into consideration of Holhoş's paper (see [15]), it can be written

$$
|f(t)-f(x)| \leq\left(1+\frac{\left(e^{-x}-e^{-t}\right)^{2}}{\delta^{2}}\right) \omega^{*}(f, \delta), \quad \delta>0 .
$$

For more details on $\omega^{*}(\cdot, \delta)$, we would like to give a reference [15] to the reader. Trivially we can write,

$$
|h(t ; x)| \leq\left(1+\frac{\left(e^{-x}-e^{-t}\right)^{2}}{\delta^{2}}\right) \omega^{*}\left(f^{\prime \prime}, \delta\right) .
$$

And also using the fact that,

$$
|h(t ; x)| \leq\left\{\begin{array}{cc}
2 \omega^{*}\left(f^{\prime \prime}, \delta\right) & , \quad\left|e^{-x}-e^{-t}\right|<\delta \\
2\left(\frac{\left(e^{-x}-e^{-t}\right)^{2}}{\delta^{2}}\right) \omega^{*}\left(f^{\prime \prime}, \delta\right) & , \quad\left|e^{-x}-e^{-t}\right| \geq \delta,
\end{array}\right.
$$

we arrive at

$$
|h(t ; x)| \leq 2\left(1+\frac{\left(e^{-x}-e^{-t}\right)^{2}}{\delta^{2}}\right) \omega^{*}\left(f^{\prime \prime}, \delta\right) .
$$

Applying Cauchy-Schwarz inequality, we obtain

$$
\begin{aligned}
& \left|n\left(M_{n}^{\alpha, \beta} h(t, x)(t-x)^{2} ; x\right)\right| \\
\leq & 2 n \omega^{*}\left(f^{\prime \prime}, \delta\right) \mu_{n, 2}^{\alpha, \beta}(x)^{\alpha}(x)+\frac{2 n}{\delta^{2}} \omega^{*}\left(f^{\prime \prime}, \delta\right) \sqrt{M_{n}^{\alpha, \beta}\left(\left(e^{-x}-e^{-t}\right)^{4} ; x\right)} \sqrt{\mu_{n, 4}^{\alpha, \beta}(x)} .
\end{aligned}
$$


Lastly, choosing $\delta=\frac{1}{\sqrt{n}}$ and with some simple calculations yield

$$
\begin{aligned}
& \left|n\left[M_{n}^{\alpha, \beta}(f ; x)-f(x)\right]+a x(x+2) f^{\prime}(x)-\frac{x(x+2)}{2} f^{\prime \prime}(x)\right| \\
\leq & \left|p_{n}\right|\left|f^{\prime}(x)\right|+\left|q_{n}\right|\left|f^{\prime \prime}(x)\right|+2 \omega^{*}\left(f^{\prime \prime}, \frac{1}{\sqrt{n}}\right)\left(2 q_{n}+x(x+2)+r_{n}\right) .
\end{aligned}
$$

Hence, the proof is completed.

Corollary 4.1. Let $f, f^{\prime \prime} \in C^{*}[0, \infty)$. Then the inequality

$$
\lim _{n \rightarrow \infty} n\left[M_{n}^{\alpha, \beta}(f ; x)-f(x)\right]=-a x(x+2) f^{\prime}(x)+\frac{x(x+2)}{2} f^{\prime \prime}(x)
$$

holds for any $x \in[0, \infty)$.

Corollary 4.2. Let $f \in C^{2}[0, \infty)$ be an decreasing and convex function. Then there exists $n_{0} \in \mathbb{N}$ such that for $n \geq n_{0}$, we have $f(x)<M_{n}^{\alpha, \beta}(f ; x)$ for all $x \in[0, \infty)$.

Presently, we are looking for the behavior of the operators on some weighted spaces. As reported in Gadziev's paper [10], set $\varphi(x)=1+e^{2 a x}, x \in \mathbb{R}^{+}$and turn the following weighted spaces over in our mind:

$$
\begin{aligned}
& B_{\varphi}\left(\mathbb{R}^{+}\right)=\left\{f: \mathbb{R}^{+} \rightarrow \mathbb{R}:|f(x)| \leq M_{f} \varphi(x), x \geq 0\right\} \\
& C_{\varphi}\left(\mathbb{R}^{+}\right)=\left\{C\left(\mathbb{R}^{+}\right) \cap B_{\varphi}\left(\mathbb{R}^{+}\right)\right\} \\
& C_{\varphi}^{k}\left(\mathbb{R}^{+}\right)=\left\{f \in C_{\varphi}\left(\mathbb{R}^{+}\right): \lim _{x \rightarrow \infty} \frac{f(x)}{\varphi(x)}=k_{f}\right\}
\end{aligned}
$$

where $M_{f}, k_{f}$ are constants depending on $f$. All three spaces are normed spaces with the norm

$$
\|f\|_{\varphi}=\sup _{x \in \mathbb{R}^{+}} \frac{|f(x)|}{\varphi(x)} .
$$

For any $f \in C_{\varphi}\left(\mathbb{R}^{+}\right)$, the inequality

$$
\left\|M_{n}^{\alpha, \beta}(f)\right\|_{\varphi} \leq\|f\|_{\varphi}
$$

holds and we complete that $M_{n}^{\alpha, \beta}(f) \operatorname{maps} C_{\varphi}\left(\mathbb{R}^{+}\right)$to $C_{\varphi}\left(\mathbb{R}^{+}\right)$.

Theorem 4.3. For each function $f \in C_{\varphi}^{k}\left(\mathbb{R}^{+}\right)$

$$
\lim _{n \rightarrow \infty}\left\|M_{n}^{\alpha, \beta}(f)-f\right\|_{\varphi}=0 .
$$

Proof. Using the general result shown in [10], the following three conditional approximations are sufficient.

$$
\lim _{n \rightarrow \infty}\left\|M_{n}^{\alpha, \beta}\left(e^{\nu a \cdot}\right)-e^{\nu a \cdot}\right\|_{\varphi}=0, \quad \nu=0,1,2 .
$$

We know that for the given operator which is represented with $M_{n}^{\alpha, \beta}, M_{n}^{\alpha, \beta}\left(e_{0}\right)=1$ and $M_{n}^{\alpha, \beta}\left(e^{2 a t}\right)=e^{2 a x}$ occurs. Presently, if we take into consideration the situation for $\nu=1$

$$
M_{n}^{\alpha, \beta}(f ; x)=n \sum_{k=0}^{\infty}\left(\begin{array}{c}
n+k-1 \\
k
\end{array}\right) \frac{\left(\theta_{n}(x)\right)^{k}}{\left(1+\theta_{n}(x)\right)^{n+k}} \int_{0}^{\infty} e^{-n t} \frac{(n t)^{k}}{k !} e^{a(n t+\alpha) / n+\beta} d t
$$

and also on the assumption that the simple calculations are made, we reach

$$
M_{n}^{\alpha, \beta}\left(e^{a t}\right)=\frac{n+\beta}{n+\beta-a} e^{a \alpha / n+\beta}\left(1-\frac{-a \theta_{n}(x)}{n+\beta-a}\right)^{-n} .
$$


Keeping an account of $\theta_{n}(x)$ and computing (4.9) with Maple,

$$
\begin{aligned}
M_{n}^{\alpha, \beta}\left(e^{a t}\right) & =e^{a x}+\frac{e^{a x}\left(-a x^{2}-\frac{4 a^{2} x^{2}}{8}-a \alpha-a\right)+a \alpha e^{a x}+a e^{a x}}{n}+\mathcal{O}\left(\frac{1}{n^{2}}\right) \\
& =e^{a x}-\frac{e^{a x} a^{2} x(x+2)}{2 n}+\mathcal{O}\left(\frac{1}{n^{2}}\right) .
\end{aligned}
$$

Conclusively,

and

$$
M_{n}^{\alpha, \beta}\left(e^{a t}\right)-e^{a x}=-\frac{e^{a x} a^{2} x(x+2)}{2 n}+\mathcal{O}\left(\frac{1}{n^{2}}\right)
$$

$$
\frac{M_{n}^{\alpha, \beta}\left(e^{a t}\right)-e^{a x}}{1+e^{2 a x}}=\frac{-a^{2} x(x+2) e^{a x}}{2 n\left(1+e^{2 a x}\right)}+\mathcal{O}\left(\frac{1}{n^{2}}\right) .
$$

And this circumstance guarantees uniform continuity. Since $M_{n}^{\alpha, \beta}\left(e_{0}\right)=1$ and $M_{n}^{\alpha, \beta}\left(e^{2 a t}\right)=$ $e^{2 a x}$, the conditions (4.8) are implemented for $\nu=0$ and $\nu=2$. Hence, the proof is completed.

Now, we desire to demonstrate that our modified operators approximate better than classical Baskakov-Szász-Stancu operators. This part, we take into consideration of article which is Aral et al [3]. Last theorem which would like to be given as below:

Theorem 4.4. Let $f \in C^{2}[0, \infty)$. Assume that there exists $n_{0} \in \mathbb{N}$, such that

$$
f(x) \leq M_{n}^{\alpha, \beta}(f ; x) \leq B_{n}^{\alpha, \beta}(f ; x), \text { for all } n \geq n_{0}, x \in(0, \infty) .
$$

Then

$$
\frac{x(x+2)}{2} f^{\prime \prime}(x) \geq\left(a x^{2}+2 a x+1\right) f^{\prime}(x) \geq 0, x \in(0, \infty) .
$$

Particularly $f^{\prime}(x) \geq 0$ and $f^{\prime \prime}(x) \geq 0$.

Contrarily, if (4.11) holds with strict inequalities at a given point $x \in(0, \infty)$, there exists $n_{0} \in \mathbb{N}$ such that for $n \geq n_{0}$

$$
f(x)<M_{n}^{\alpha, \beta}(f ; x)<B_{n}^{\alpha, \beta}(f ; x) .
$$

Proof. From (4.10) we have that

$$
0 \leq n\left(M_{n}^{\alpha, \beta}(f ; x)-f(x)\right) \leq n\left(B_{n}^{\alpha, \beta}(f ; x)-f(x)\right), \text { for all } n \geq n_{0}, x \in(0, \infty) .
$$

Considering an asymptotic formula which is held by classical Baskakov-Szász-Stancu operators [17],

$$
\lim _{n \rightarrow \infty} n\left(B_{n}^{\alpha, \beta}(f ; x)-f(x)\right)=(1+\alpha-\beta x) f^{\prime}(x)+\frac{x(x+2)}{2} f^{\prime \prime}(x) .
$$

Thanks to (4.10), we also accept that $1+\alpha-\beta x \geq 0$. Combining (4.7) and (4.12)

$$
0 \leq\left(a x^{2}+2 a x+1+\alpha-\beta x\right) f^{\prime}(x) \leq \frac{x(x+2)}{2} f^{\prime \prime}(x),
$$

we can reach (4.11) easily.

Contrarily, if (4.11) holds with strict inequalities for a given $x \in(0, \infty)$ then

$$
0<\left(a x^{2}+2 a x+1+\alpha-\beta x\right) f^{\prime}(x)<\frac{x(x+2)}{2} f^{\prime \prime}(x)
$$

employing asymptotic formulas for modified operators (4.7) and the classical operators (4.12), we have the desired result. 


\title{
REFERENCES
}

[1] T. Acar, A. Aral, H. Gonska: On Szász-Mirakyan operators preserving e $e^{2 a x}, a>0$. Mediterr. J. Math. 14 (1) (2017), Art. 6, 14 pp.

[2] T. Acar, A. Aral, D. Cárdenas-Morales, P. Garrancho: Szász-Mirakyan type operators which fix exponentials. Results Math. 72 (2) (2017), no. 3, 1393-1404.

[3] A. Aral, D. Cárdenas-Morales, P. Garrancho: Bernstein-type operators that reproduce exponential functions. J. Math. Inequal.(Accepted).

[4] M. Birou: A note about some general King-type operators. Ann. Tiberiu Popoviciu Semin. Funct. Equ. Approx. Convexity, 12 (2014), 3-16.

[5] P. I. Braica, L. I. Pişcoran, A. Indrea: Grafical structure of some King type operators. Acta Universitatis Apulensis. 34 (2013), 163-171.

[6] D. Cárdenas-Morales, P. Garrancho, F. J. Munoz-Delgado: Shape preserving approximation by Bernstein-type operators which fix polynomials. Appl. Math. and Comput. 182 (2) (2006), 1615-1622.

[7] D. Cárdenas-Morales, P. Garrancho, I. Raşa: Approximation properties of Bernstein-Durrmeyer type operators. Appl. Math. Comput., 232 (2014), pp. 1-8.

[8] E. Deniz, A. Aral, V. Gupta: Note on Szász-Mirakyan-Durrmeyer operators preserving $e^{2 a x}, a>0$. Numer. Funct. Anal. Optim. 39 (2) (2018), 201-207.

[9] O. Duman, M. A. Özarslan: Szász- Mirakyan type operators providing a better error estimation. Appl. Math. Lett. 20 (2007) 1184-1188.

[10] A. D. Gadziev: Theorems of the type of P. P. Korovkin's theorems. Mat Zametki 20 (5), (1976), 781-786.

[11] V. Gupta, G.S. Srivastava: Simultaneous approximation by Baskakov-Szász type operators. Bull. Math. Soc. Sci. 37 (85) (1993), 73-85.

[12] V. Gupta, A. M. Acu: On Baskakov-Szász-Mirakyan type operators preserving exponential type functions. Positivity. pp 1-11, doi:10.1007/s11117-018-0553-x.

[13] V. Gupta, A. Aral: A note on Szász-Mirakyan-Kantorovich type operators preserving $e^{-x}$. Positivity. 22, pp 415-423, doi:10.1007/s11117-017-0518-5.

[14] V. Gupta, G. Tachev: On approximation properties of Phillips operators preserving exponential functions. Mediterr. J. Math. 14 (4) (2017), Art. 177, pp. 12.

[15] A. Holhoş: The rate of approximation of functions in an infinite interval by positive linear operators. Studia Univ. BabesBolyai Mathematica. 55 (2) (2010), pp. 133-142.

[16] J. P. King: Positive linear operators which preserve $x^{2}$. Acta Math. Hungar. 99 (3) (2003) 203-208.

[17] V. N. Mishra, M. Mursaleen, P. Sharma: Some approximation properties of Baskakov-Szász-Stancu operators. Appl. Math. Inf. Sci. 9 (6) (2015), 3159-3167.

[18] Ö. G. Yılmaz, A. Aral, F. Taşdelen Yeşildal: On Szász-Mirakyan type operators preserving polynomials. J. Numer. Anal. Approx. Theory 46 (1) (2017), 93-106.

[19] Ö. G. Yılmaz, V. Gupta, A. Aral: On Baskakov operators preserving the exponential function. J. Numer. Anal. Approx. Theory 46 (2) (2017), 150-161.

\author{
ANKARA UNIVERSITY \\ DEPARTMENT OF MATHEMATICS \\ 06100, ANKARA, TURKEY \\ E-mail address: bodureankara.edu.tr \\ ANKARA UNIVERSITY \\ DEPARTMENT OF MATHEMATICS \\ 06100, ANKARA, TURKEY \\ E-mail address: ogurel@ankara.edu.tr \\ KIRIKKALE UNIVERSITY \\ DEPARTMENT OF MATHEMATICS \\ 71450, KIRIKKALE, TURKEY \\ E-mail address: aliaral73@yahoo.com
}

